\title{
Assessing the Feasibility of the Spectrum Sharing Concepts for Private Industrial Networks Operating above $5 \mathrm{GHz}$
}

\author{
Pekka Ojanen ${ }^{1}$, Seppo Yrjölä ${ }^{2}$, Marja Matinmikko-Blue ${ }^{3}$ \\ ${ }^{1}$ Co-Worker Technology Finland Oy, Turku, Finland, pekka.ojanen@co-workertech.com \\ ${ }^{2}$ Nokia, Oulu, Finland \\ ${ }^{3}$ University of Oulu, Oulu, Finland
}

\begin{abstract}
Ongoing fifth generation mobile network 5G deployment is bringing higher speeds, higher capacity, lower latency and greater reliability into connectivity enabling data sharing amongst participating components of industrial systems. The private industrial network opportunity for serving different verticals is largely dependent on the timely availability, quality and the cost of spectrum. The growing pressure to open the wireless market for location specific networks has resulted in new regional licensing and sharingbased models for spectrum management. This paper discusses private industrial network requirements for the spectrum management through a framework that can be used to assess the feasibility of the spectrum management approaches. Specifically, recent sharing concepts above $5 \mathrm{GHz}$ in the US, Europe and four selected countries: Australia, Hong Kong, Japan and $U K$ are analyzed from the viewpoint of private industrial networks. Each of the selected sharing concepts is a unique approach to make wideband spectrum available for other network providers than traditional MNO's.
\end{abstract}

Index Terms - Industrial Internet of Things, LTE, Private networks, Spectrum management, Regulation, 5G.

\section{INTRODUCTION}

The digitalization of various vertical sectors such as the manufacturing, logistics, transportation, automotive and agriculture will be built upon the upcoming 5th generation mobile network (5G) infrastructure. The next era in industrial production "Industry 4.0 " is aiming at improving significantly the flexibility, versatility, usability and efficiency of industries through integrating the internet of things (IoT) and related services [1]. In order to fully realize the benefits of upcoming $5 \mathrm{G}$ networks in serving the different vertical sectors' location specific needs, there is an urgent need to allow the verticals themselves or external service providers to operate their own $5 \mathrm{G}$ networks without direct mobile network operator (MNO) involvement [2]. While having high synergy with selected public mobile network use cases like enhanced mobile broadband, the performance and operational connectivity requirements of the industrial use cases may be significantly different, particularly related to network availability, security and privacy [3]. If an industrial or a private network is deployed by an organization other than current mobile license holder, e.g., by a micro-operator [4], the organization needs to get access to suitable spectrum stressing the role of the spectrum management [5][6].

As the spectrum demand is changing towards wider bandwidths to facilitate higher network capacities and higher bitrate services, traditional private mobile radio (PMR) systems providing voice and narrowband data have been widely replaced by wireless local area networks (RLANs) in industry environment. The utilization of unlicensed bands may have limitations in coverage, mobility and quality of service (QoS) depending on the application. Instead, employment of standardized wide area mobile technologies, such as third generation partnership project (3GPP) defined Long Term Evolution (LTE), and emerging 5G could better meet the performance requirements of industrial networks [7]. To date, most LTE bands have been made available for nationwide public mobile networks through competitive awarding, i.e., auctions [8], and the same approach has dominated the $5 \mathrm{G}$ spectrum release so far [9]. Only a few countries have introduced regulatory frameworks for making spectrum available for individually authorized, locally deployed private networks. As one solution to overcome the spectrum scarcity, variants of 3GPP technologies have been developed suitable for deployment in the license exempt bands.

Suitable regulatory frameworks for local 5G deployments were studied in [10], more detailed studies on locally and temporary shared spectrum are in [11] and micro licensing and the associated regulatory framework in [12]. Reference [13] proposed a method to assess spectrum management approaches in the context of locally deployed industrial IoT networks and analyzed recent approaches below $5 \mathrm{GHz}$ in Canada, Finland, France, Germany, Netherlands, UK and US.

This paper aims to expand the analysis to spectrum bands above $5 \mathrm{GHz}$ and examines recent spectrum management approaches facilitating deployment and operation of private industrial networks to meet the requirements towards the $5 \mathrm{G}$ era. The focus is on use cases where private networks are deployed by industrial organizations for their own use and thus, they need to get access to suitable spectrum on 
affordable terms. Information on studied spectrum management approaches have been collected both from public sources and directly from the regulators. This research addresses the planning and authorization processes which are key parts of the overall spectrum management process [14].

The rest of the paper is organized as follows. In Section II, spectrum and regulatory requirements are discussed, and assessment framework introduced. Next, selected recent spectrum management approaches relevant for private industrial networks are described, assessed and regulatory recommendation given. Conclusions are drawn in section IV.

\section{REQUIREMENTS OF PRIVATE INDUSTRIAL NETWORKS}

Use cases of vertical industries and their functional requirements related to communication services [7] have an impact on preferred spectrum management approach. High service availability can be reached through exclusive access to dedicated spectrum assignments and through protection from harmful interference. The required service areas are distinct and regional, ranging from local indoor coverage, up to few $\mathrm{km}^{2}$. For higher frequencies above $5 \mathrm{GHz}$ signal attenuation can significantly limit the coverage area. Depending on the application, access to wide bandwidths is needed in either direction requiring uplink/downlink ratio (UL/DL) flexibility from the technology and the band regulation. Several industry applications like automated guided vehicles (AGVs) and drones need high mobility offered particularly by mobile technologies like LTE and 5G. Time division duplex (TDD) technology widely deployed at high frequencies may need to be synchronized with adjacent networks, which would limit the flexibility and applicability. In order to meet extremely demanding latency and reliability requirements, licensed spectrum and protection from harmful interference are highly preferred. Due to the critical time-toair and shorter investment cycles, vertical industries may prefer to deploy their own dedicated networks and require the assurance on the continuity of service, without unjustified price increases, spectrum re-farming or technology upgrades over the planned life span. Moreover, the license cost, duration and overall regulatory certainty should be comparable to the investment cycle, the suitable authorization process would be application based, and the applications submission based on the business timing. The key elements of the proposed spectrum management assessment approach and the preferences are identified and summarized in Table I.

\section{TABLE I. SHARED SPECTRUM ASESSMENT FrAMEWORK}

\begin{tabular}{ll}
$\begin{array}{l}\text { Management } \\
\text { framework element }\end{array}$ & Private industrial network preference \\
\hline Band type & Harmonized bands for economies of scale. \\
Bandwidth & Sufficient for wideband applications. \\
Spectrum availability & High availability, guaranteed.
\end{tabular}

Interference protection By regulator, authorization conditions or technical solution.

Sharing conditions Stable, pre-defined.

Mobility Support for mobile use cases.

Location and coverage Applicant defined: outdoor local or area regional, or indoor as required by the use case. One or multiple areas.

Maximum transmit Sufficient for required local/regional power outdoor and/or indoor coverage.

\section{UL/DL ratio Flexible}

Authorization method Application submission any time, time-toair critical.

Authorization In line with investment cycle and business duration

Cost/pricing case.

Fee, administrative one-off or annual fee, per license/area.

Regulatory certainty High, beyond authorization duration.

\section{Shared SPECTRUM APPROACHES FOR PRIVATE INDUSTRIAL NETWORKS ABOVE $5 \mathrm{GHZ}$}

Both in the US and in Europe, there are studies ongoing on the possibilities to make $6 \mathrm{GHz}$ spectrum available for RLAN-type and other license exempt use to complement 5 $\mathrm{GHz}$ wireless access systems in the WAS/RLAN bands used as a reference case in this study. Additionally, several countries are in the process of making shared spectrum available in the 26 or $28 \mathrm{GHz}$ range for private or industrial applications. These bands are selected for the study due to their timely relevance as being examples of bands planned for shared spectrum access in different regions of the world.

\section{A. Selected Shared Spectrum Approaches}

\section{1) The $5 \mathrm{GHz}$ RLAN Bands (5G RLAN)}

The Radiocommunication Sector of the International Telecommunication Union (ITU-R) has identified the bands $5150-5350 \mathrm{MHz}$ and $5470-5725 \mathrm{MHz}$ globally for WASs, including RLANs. Several incumbent applications are deployed in those bands, and the deployment of WASs must not cause harmful interference to the incumbent applications. Therefore, a number of technical and operational restrictions have been defined, such as maximum transmit power, restriction to indoor use in part of the bands, restrictions on antenna pattern [15] as well as required interference mitigation methods, such as dynamic frequency selection (DFS) and transmit power control (TPC) [16]. The listenbefore-talk protocol (LBT) may also need to be employed to facilitate coexistence both with WASs sharing the band [17]. RLAN Systems compliant with the ETSI standard [17] are allowed to be deployed in the WAS/RLAN bands within the EU and the CEPT countries, as well as in many other countries outside Europe. The FCC in the US has defined their technical requirements for deployment and operation of U-NII devices in the WAS/RLAN bands, and also in the 
5725-5850 MHz [18], applicable in several other countries as well.

LTE-U and MulteFire [19] are LTE based technologies that are designed to be compliant with the regulatory requirements in the $5 \mathrm{GHz}$ bands. WAS/RLAN networks can in most countries be deployed in the $5 \mathrm{GHz}$ bands under a general authorization, and the same applies also to compliant LTE based $5 \mathrm{GHz}$ networks for private and industrial applications.

\section{2) RLAN Extension to the $6 \mathrm{GHz}$ Bands}

Due to the success of RLAN and the increasing use of license exempt frequencies, the FCC is preparing for making additional spectrum available for U-NII devices and the CEPT for WAS/RLAN in the frequencies above the $5 \mathrm{GHz}$.

The FCC addresses the $1200 \mathrm{MHz}$ of spectrum in the 5.925-7.125 GHz as the $6 \mathrm{GHz}$ band [20]. Several incumbent services are operating in the band. The proposed technical requirements are rather similar to those for U-NII devices in the $5 \mathrm{GHz}$ bands, aiming to protect the incumbents from harmful interference. The $6 \mathrm{GHz}$ band is divided into 4 subbands, two of them (U-NII-5 and U-NII-7) are to be allowed for standard-power access points operating under the control of an Automated Frequency Coordination (AFC) system. The AFC is a system that automatically determines and provides lists of which frequencies are available for use by access points. The maximum conducted output power is 1 W. The other two sub-bands (U-NII-6 and U-NII-8) are to be allowed for low-power access points. The maximum conducted output power is $250 \mathrm{~mW}$. For client devices the maximum conducted output power is $63 \mathrm{~mW}$.

The CEPT is studying the feasibility of making the band 5925-6425 MHz available for WASs including RLANs. Initial technical studies addressed sharing and coexistence with the in-band and adjacent band incumbents. Based on the studies the CEPT expects that compatibility and coexistence between WAS/RLAN and existing services within and adjacent to the band 5925-6425 $\mathrm{MHz}$ be technically feasible under certain conditions. Requirements for indoor operation, limited output power, use of guard bands and requirements for out-of-band emissions would make the incumbent protection feasible. The CEPT will also look into innovative sharing solutions for geographical protection of incumbents. The aim is to make an ECC Decision in 2020, which would facilitate the WAS/RLAN deployment in the $6 \mathrm{GHz}$ in the CEPT countries.

The $6 \mathrm{GHz}$ band is not on the agenda of the WRC-19, and thus it's identification for IMT is not expected.

\section{3) The $28 \mathrm{GHz}$ Shared Spectrum band in Hong Kong}

The frequencies 27.95-28.35 GHz were made available in July 2019 as four $100 \mathrm{MHz}$ channels on a geographically shared basis for the provision of wireless broadband services on a local basis for specific groups of users based on the $5 \mathrm{G}$ or other advanced mobile technologies [21]. The assignments will be made on a first-come-first-served basis under a new Localized Wireless Broadband Service (LWBS) license type with less stringent requirements as compared with conventional public mobile services licenses. There is no limit for LWBS licenses to be issued. The license duration is 5 years and may be extended for further 5 years [22].

The applicant defines the required network deployment, including coverage areas, the maximum total area being 50 $\mathrm{km}^{2}$. Assignment of the Shared Spectrum to each of the successful applicants will be subject to a spectrum cap of 400 MHz. The actual amount to be assigned will be decided by the regulator, based on the merits of the application. Deployments in specific locations are foreseen, such as university campuses, industrial facilities, airport, technology villages, etc. Real time voice communications to and from PSTN is not allowed, and normally the assignees will not be allocated mobile subscriber numbers from the numbering plan of Hong Kong. There are fee based costs, comprising of annual license fee and possible spectrum utilization fee (SUF), to be paid if the spectrum becomes congested. The annual license fee has a fixed part, and additionally depends on the number of devices and base stations and spectrum amount. The licensees shall coordinate and agree with other licensees on the technical measures to be taken to minimize any mutual interference between them.

The $28 \mathrm{GHz}$ band is not an IMT candidate band at the coming WRC-19.

\section{4) The $28 \mathrm{GHz}$ Band in Japan}

In Japan, an initiative called local 5G aims at making spectrum available for specific applications, for vertical sector players. The band $28.2-28.3 \mathrm{GHz}$ is to be made available first. The aim is to make the band $28.3-29.1 \mathrm{GHz}$ available later, in late 2020, when studies on the sharing conditions with satellite incumbents are completed. The licenses in the 28.2-28.3 GHz will be granted to the owners of the land or property covered in the application. If the land or building is not owned by the applicant, then the service is limited to fixed wireless. Existing mobile operators are not allowed to apply for local $5 \mathrm{G}$ licenses.[23]

\section{5) The $26 \mathrm{GHz}$ Shared Access Band in the UK}

The UK regulator Ofcom is making spectrum in four Shared Access bands available through local licenses, one of the bands being $26 \mathrm{GHz}(24.25-26.5 \mathrm{GHz})$. The band will be available for $5 \mathrm{G}$ indoor deployments. The regulatory framework is already defined, and the licensing window will open by the end of 2019. There are two types of licenses, low power license (per area license) and medium power license. The low power license allows the users to deploy a required number of base stations in a circular area with a $50 \mathrm{~m}$ radius, terminals are covered by the same license. For larger areas, people can apply for multiple licenses to achieve the required coverage. Medium power licenses are only for deployments in rural areas issued on a per base station basis. The licenses are assigned on a first-come, first served basis and the access is coordinated by Ofcom to ensure avoidance of harmful interference between the users. The licenses are technology neutral, but Ofcom has determined technical license conditions for the band, covering e.g., permitted deployment including antenna height, authorized bandwidth, maximum 
transmit power for base stations and terminals, emission limits and out-of-band emission limits. The maximum total radiated power (TRP) for base stations is $200 \mathrm{~mW} / 200 \mathrm{MHz}$ and for terminals $200 \mathrm{~mW}$. Authorized bandwidths can be 50,100 or $200 \mathrm{MHz}$. The licenses are valid for an indefinite duration. The annual license fee reflects Ofcom's cost of issuing the license, being fixed $£ 320$ for the $26 \mathrm{GHz}$ band. [24][25]

\section{6) The $26 \mathrm{GHz}$ Band in Australia}

The regulator ACMA plans to make the $26 \mathrm{GHz}$ band (24.25-27.5 GHz) available for fixed point-to-multipoint or mobile network deployments around the end of year 2020 [26],[27]. The sub-band $24.25-24.7 \mathrm{GHz}$ is to be made available Australia-wide, through LIPD class license for wireless broadband limited to private property (including domestic, industrial, business, commercial and government use cases) and restricted to indoor use only. Class licenses do not provide protection from interference. The maximum TRP of the base stations is $100 \mathrm{~mW} / 200 \mathrm{MHz}$ and for user terminals the maximum TRP is $158 \mathrm{~mW}$.

The sub-band $24.7-25.1 \mathrm{GHz}$ is to be made available also Australia-wide, through apparatus licensing for wireless broadband, limited to private property as in the a.m. band, but both for indoor and outdoor use. The maximum license bandwidth is either 100 or $150 \mathrm{MHz}$, depending on the frequency, and the maximum transmit powers are somewhat higher than for class licensed case.

The band $25.1-27.5 \mathrm{GHz}$ is to be made available as 100 $\mathrm{MHz}$ blocks in 34 metropolitan areas and regional centers through spectrum licensing for wide-area wireless broadband and elsewhere through apparatus licensing (potentially using a new spectrum-space apparatus license type) for wireless broadband. The spectrum licenses are proposed to be auctioned. The maximum bandwidth for the apparatus licenses is $400 \mathrm{MHz}$. Existing incumbents will be able to continue to operate with license conditions included on new wireless broadband licenses that ensure coexistence. For the 25.1-27 GHz band additional proposed license conditions are to be applied to protect specific incumbents.

\section{B. Spectrum Management Assessment and Discussion}

The summary of the assessment of the selected spectrum management approaches is depicted in Table II based on the assessment framework from section II. The assessment results show differences between the spectrum management approaches selected by the different regulators in the considered band. Based on these assessment results, several recommendations can be drawn. The heterogeneity of industrial use cases, applications and requirements leads to a flexibility requirement in spectrum award and use. For mission critical applications, and applications requiring high spectrum availability and quality, individual local authorizations and protection from harmful interference are required. The authorizations should be based on applications, and submissions should be allowed any time, based on the business need.
TABLE II. ASSESSMENT OF SHARED SPECTRUM APPROACHES

\begin{tabular}{|c|c|c|c|c|c|c|}
\hline $\begin{array}{l}\text { Shared Spectrum } \\
\text { management } \\
\text { framework } \\
\text { element }\end{array}$ & 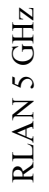 & $\begin{array}{l}\stackrel{N}{J} \\
0 \\
Z \\
\mathbb{Z} \\
\vec{Z}\end{array}$ & $\begin{array}{l}\underset{N}{N} \\
\underset{D}{N} \\
\stackrel{v}{I}\end{array}$ & 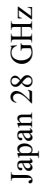 & 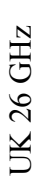 & 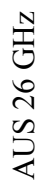 \\
\hline Band type & + & + & - & - & + & + \\
\hline Bandwidth & + & + & + & - & + & + \\
\hline Spectrum availability & - & - & - & + & + & - \\
\hline Interference protection & - & - & - & + & + & - \\
\hline Sharing conditions & - & + & - & + & + & + \\
\hline Mobility & + & + & - & - & - & - \\
\hline Location and coverage & + & - & + & + & - & - \\
\hline Max. transmit power & + & - & + & $?$ & + & + \\
\hline $\mathrm{UL} / \mathrm{DL}$ ratio & + & + & + & + & + & + \\
\hline Authorization method & + & + & + & $?$ & + & + \\
\hline Authorization duration & + & + & + & $?$ & + & + \\
\hline Cost/pricing & + & + & - & - & - & + \\
\hline Regulatory certainty & + & - & + & - & + & - \\
\hline
\end{tabular}

The applicants should be allowed to define the required bandwidth and coverage area. In case licensing is used, the preferred license duration should be aligned with the licensee's investment cycle. For industrial networks 10 years would be desirable and the license should be renewable. Only reasonable administrative fees should be applied, on a yearly basis or per transmitter where practical. Spectrum should be made available from harmonized 3GPP bands, for most cases preferably on exclusive basis in the coverage area resulting in spectrum sharing in geographical dimension. In all cases indoor and outdoor network deployment should be allowed and the maximum transmit power should be sufficient for the planned coverage. Implementation and employment of complex mitigation methods should not be required. UL/DL asymmetry should be facilitated through employment of TDD duplexing, and network synchronization should be required only in locations where it is required for coexistence reasons. Where synchronization is needed, the UL/DL ratio should be defined in a manner that considers the requirements of all affected networks.

Utilization of the $5 \mathrm{GHz}$ bands with better propagation characteristics and mobile 3GPP technologies enables best the mobile industrial applications. The $5.8 \mathrm{GHz}$ band offers also the highest output power of $4 \mathrm{~W}$. $6 \mathrm{GHz}$ RLAN will offer up to $1.2 \mathrm{GHz}$ extension to highly occupied $5 \mathrm{GHz}$ spectrum and is new 3GPP band for the 5G NR-U offering economies of scale. In Hong Kong the utility of the $28 \mathrm{GHz}$ band is limited by the interference coordination between licensees. The land ownership in Japan provides eligibility to access the $28 \mathrm{GHz}$ spectrum in the distinct location. Ofcom in the UK has defined a complete regulatory framework for 
$26 \mathrm{GHz}$ suitable for the industrial use cases indoors. The 26 $\mathrm{GHz}$ band in Australia is segmented for different vertical use cases but compared to the UK regulation the Australian framework does not offer coordination against harmful interference.

\section{CONCLUSIONS}

The trend to higher frequencies, above $5 \mathrm{GHz}$ with smaller radio coverages and the increasing role of indoor networks will boost sharing in cities and indoor spaces and drive the local operator paradigm. This paper has highlighted the importance of understanding the differentiating requirements and approaches for spectrum management in the context of the local industrial private networks. We have defined an assessment framework and assessed recent spectrum management approaches in the $5 \mathrm{GHz}, 6 \mathrm{GHz}, 26$ $\mathrm{GHz}$ and $28 \mathrm{GHz}$ bands in the selected countries. Results show that different countries have adopted different spectrum management approaches, which ultimately define who can enter the private industrial network market and which sub-segments and use cases can be served. These findings are supported also by the previous literature [9]. Based on the assessment, key differentiators are: band type, spectrum availability (both for facilitating the network deployment and during the operation), possibility for interference free operation and technical and operational requirements to facilitate incumbent protection. All employed authorization methods are suitable for industrial networks. On the other hand, the spectrum availability is in some cases limited to indoor use only or there is only limited or no protection against harmful interference.

Future research is needed in spectrum coexistence management to enable dense local spectrum assignments, and in pricing of spectrum to achieve transparency and inclusiveness.

\section{ACKNOWLEDGMENT}

The research was partly supported by the Business Finland 5G-VIIMA INSIDE project.

\section{REFERENCES}

[1] Federal Ministry for Economic Affairs and Energy, Platform Industrie 4.0 https://www.plattformi40.de/I40/Navigation/EN/Industrie40/WhatIsIndustrie40/what-isindustrie40.htm

[2] J. Zander, "Beyond the ultra-dense barrier: Paradigm shifts on the road beyond 1000x wireless capacity," IEEE Wireless Communications, vol. 24, no. 3, pp. 96-102, 2017

[3] 5G Alliance for Connected Industries and Automation (5G-ACIA), 5G for Connected Industries and Automation White Paper, 2018.

[4] M. Matinmikko, M. Latva-aho, P. Ahokangas, S. Yrjölä, and T. Koivumäki, "Micro operators to boost local service delivery in 5G," Wireless Personal Communications, vol. 95, no. 1, pp. 69-82, 2017.

[5] R. Ferrus and O. Sallent, "Extending the LTE/LTE-A Business Case: Mission- and Business-Critical Mobile Broadband Communications," IEEE Vehicular Technology Magazine, vol. 9, no. 3, pp. 47-55, 2014
[6] F. Beltran, "Accelerating the introduction of spectrum sharing using market-based mechanisms," IEEE Communications Standards Magazine, vol. 1, no. 3, pp. 66-72, 2017.

[7] 3rd Generation Partnership Project, 3GPP TR 22.804, V16.1.0, (Release 16), Technical Specification Group Services and System Aspects; Study on Communication for Automation in Vertical Domains, 2018.

[8] P. Cramton, "Spectrum auction design," Review of Industrial Organization, vol. 42, no. 2, pp. 161-190, 2013.

[9] M. Matinmikko-Blue, S. Yrjola, V. Seppänen, P. Ahokangas, H. Hämmäinen, and M. Latva-aho, "Analysis of Spectrum Valuation Elements for Local 5G Networks: Case Study of 3.5-GHz BandAnalysis of Spectrum Valuation Elements for Local 5G Networks: Case Study of $3.5 \mathrm{GHz}$ Band," IEEE Transactions on Cognitive Commuications and Networking, vol. 5, no. 3, pp. 741-753, 2019.

[10] European Commission Radio Spectrum Policy Group, RSPG18-005 Strategic spectrum roadmap towards $5 \mathrm{G}$ for Europe: RSPG second opinion on $5 \mathrm{G}$ networks, 2018.

[11] M.D.P. Guirao, A.Wilzeck, A. Schmidt, K. Septinus, and C. Thein, "Locally and temporary shared spectrum as opportunity for vertical sectors in 5G," IEEE Network, vol. 31, no. 6, pp. 24-31, 2017.

[12] M. Matinmikko, S. Yrjölä, and M. Latva-aho, "Micro operators for ultra-dense network deployment with network slicing and local spectrum micro licensing," The 2018 IEEE 87th Vehicular Technology Conference, Porto, 2018.

[13] P. Ojanen and S. Yrjölä, "Assessment of Spectrum Management Approaches to Private Industrial Networks," 13th International Conference Cognitive Radio Oriented Wireless Networks and Communications (CROWNCOM), Poznan, Poland, June 2019

[14] International Telecommunication Union (ITU), the ICT regulation toolkit project by ITU and the World Bank's Information for Development Program (infoDev).

[15] ITU-R, Resolution 229. Use of the bands $5150-5250 \mathrm{MHz}, 5250$ $5350 \mathrm{MHz}$ and $5470-5725 \mathrm{MHz}$ by the mobile service for the implementation of wireless access systems including radio local area networks, 2012.

[16] ITU-R, Recommendation M.1450-5. Characteristics of broadband radio local area networks, 2014

[17] ETSI, EN 301 893. 5 GHz RLAN; Harmonised Standard covering the essential requirements of article 3.2 of Directive 2014/53/EU, 2017.

[18] CFR Title 47, Part 15, Subpart E. Unlicensed National Information Infrastructure Devices.

[19] MulteFire alliance homepage, http://www.multefire.org/

[20] FCC, NPRM 18-147. Unlicensed Use of the $6 \mathrm{GHz}$ Band, 2018.

[21] OFCA Press release: https://www.ofca.gov.hk/en/media focus/press releases/index id 19 53.html

[22] OFCA, Guideline GN-13/2019. Guidelines for Submission of Applications for Assignment of Shared Spectrum in the $26 \mathrm{GHz}$ and $28 \mathrm{GHz}$ Bands, 2019.

[23] Ministry of Internal Affairs and Communications (MIC), 5th generation local $\quad 5 \mathrm{G}$, http://www.soumu.go.jp/main_sosiki/joho_tsusin/policyreports/joho_t susin/5th_generation/local_5g/index.html

[24] Ofcom, Shared Access license: guidance document, 2019.

[25] UK Interface Requirements (IR) 2105, Shared Access Indoor $26 \mathrm{GHz}$, 2019.

[26] ACMA, Future use of the $26 \mathrm{GHz}$ band - Planning decisions and preliminary views, 2019.

[27] ACMA, Draft spectrum reallocation recommendation for the $26 \mathrm{GHz}$ band in cities and regional centres - Consultation paper, 2019. 